\title{
Design and Development of a Monitoring System for Traffic Police Based on J2EE Technology \\ LI Xiao-xia
}

(Hunan Railway Professional Technology College, ZhuZhou Hunan 412001)

\author{
Key words: Traffic Police; Monitoring System; J2EE Technology
}

\begin{abstract}
This article elaborates monitoring system features, development status and technical features systematically, analyzes the needs of traffic monitoring systems and design appropriate solutions. Technologies of this system is J2EE based Java and MVC. This system considers the performance of traffic monitoring and divides the module according function. Its seven modules are user login, user management, basic imformation inquery, vehicle monitoring and display, vehicle dispatched, alarming, electronic map. This article ultimately elaborates the detailed design and implementation of the system. It had been proved by deploy and test that traffic police monitoring system based on J2EE technology and proposed in this paper greatly improves the flexibility, accuracy of blending, and efficiency of handling cases for traffic police.
\end{abstract}

\section{Introduction}

This paper in view of the problems existing in current monitoring system not to be compatible and development costs etc. design a traffic police of public security monitoring system based on J2EE technology, system contains information inquiry, vehicle monitoring display, automatic alarm, statistical information, vehicles dispatched, with user login and management, and map display function, traffic information fully shared provides strong support for the relevant departments. The most basic function of the system is user login and management, access to the system must have a legal user identity and the corresponding rights. User rights are divided into many levels, for ordinary users, generally only have the non sensitive information of the query operation, do not have the information to modify and delete operation. The full sharing of traffic information is the establishment of a unified data interface, the database is the key to achieve a unified data interface, unified data access will greatly enhance the system's performance and reduce the maintenance cost of the system. Through the network, users only need to any network in the network of the terminal nodes can access system, to obtain the corresponding information, automatic monitoring traffic state, so as to realize the "real-time monitoring, automatic alarm, rapid response" of the traffic monitoring system. This part of the public security traffic police coordination of road traffic, timely detection of traffic violations and arrested the suspect vehicle has brought great benefits

\section{J2EE structural framework}

J2EE according to the function of the application logic is divided into a number of components, these components according to the level of functional distribution in each machine, so as to form a Distributed Multi-level architecture. J2EE appear before, Web application is the most client / server model, this model requires a higher client, each deployment requires a lot of resources, the system upgrade and expansion is relatively difficult. J2EE introduction is in order to solve the shortcomings the client / server, the application system into a multi-layer model, various service functions belong to a separate layer, which for the system upgrade and expansion is very useful. Generally speaking, the J2EE framework has the following four layers.

(1) EIS layer, running on the J2EE server;

(2) the business logic layer, running on the J2EE server;

(3) Web layer, running on the J2EE server;

(4) the client layer, running on the client side; 
The first is the customer layer, which directly runs on the client, and the server for communication. As shown in Fig. 1, it shows the customer layer composition and the server communication schematic. When the user browser or application access to the client, it is necessary to consider the function of the load is to choose the client or server, the general need to choose a balance point. The principle of selecting the balance point is that if the function is placed on the server, the distributed performance of the system is stronger, and it is easier to manage. If the function is loaded by the client, the distributed performance is relatively poor, but the user experience will be better. The dependence of these two methods on network performance is alsodifferent.

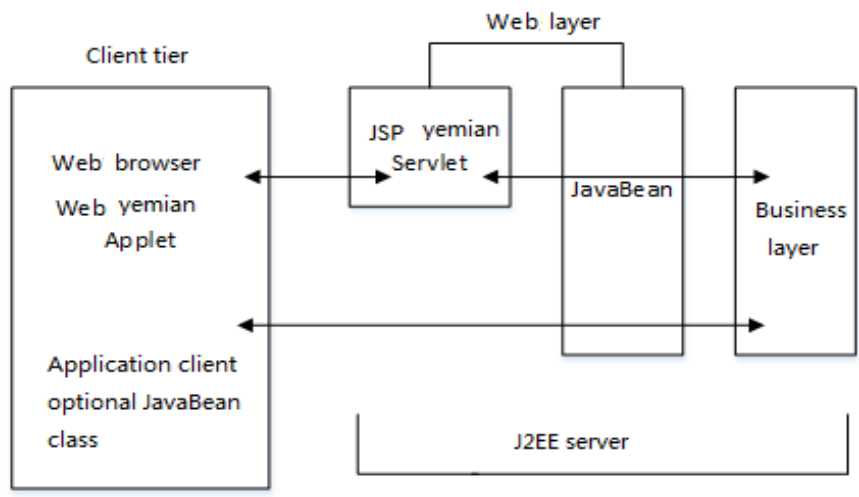

Fig.1 J2EE customer interaction schematic diagram

Web layer is mainly to provide the support of the customer layer, running on the server. In general, static page is not a web component, web layer is generally for dynamic interactive web page, also contains the many objects to accept the user data and the relevant data to the upper logic to deal with.

Fig. 2 is a schematic diagram of the Web layer interaction.

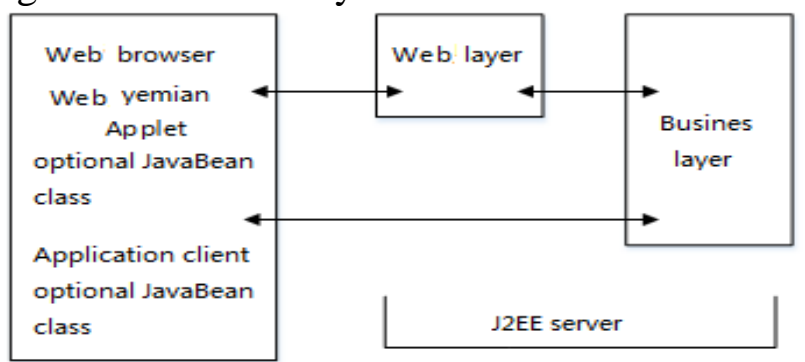

Fig. 2 Schematic diagram of Web layer interaction

Business layer is mainly for the specific business logic processing, running on the server, generally speaking, the business layer on the processing. Fig.3 shows that a client program receives information and processes the corresponding processing, and sends it to the EIS layer for storage. The process is reversible.

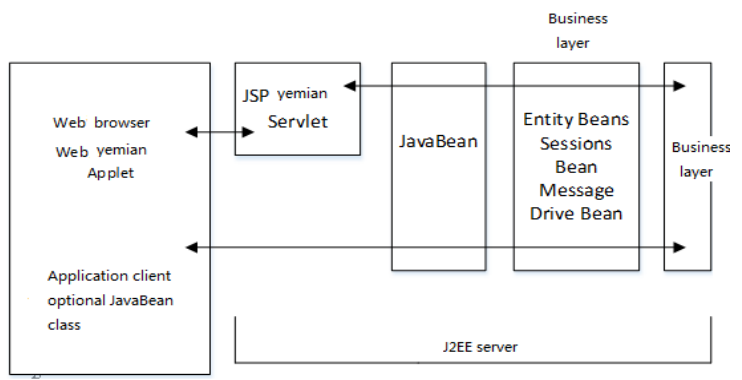

Fig.3 business logic layer

J2EE framework with three kinds of EJB, namely Bean Enitity, Beans Message and driven Beans Session. Where Bean Session represents a temporary interaction between the client and the client, and the life cycle is accompanied by the execution of the client. While the Beans Enitity is a permanent data record, generally stored in the database. At the end of the session, there will be a mechanism to ensure that the data is stored in the Beans Enitity data. Message driven beans in the 
session bean into the message listener characteristics, combined with the JMS (JavaMessage service message listener, allowing a business component layer asynchronous receive JMS message.

\section{Struts+Hibernate+Spring}

MVC was originally used to build the user interface in Smalltalk-80. The purpose of the introduction is to reduce the coupling between application code and data representation and description in order to make the code reusable. The flexibility, expansibility and maintainability of the software are greatly improved.

In order to improve the specification of distributed system development, data model and access control data separation strategy can be adopted in general. MVC also uses a similar strategy,

The design pattern is composed of three parts: model, view and controller. Among them, controller is used to control the corresponding behavior of the client for the input, and the corresponding action of the user is converted to the specific operation of the model. View is the client's display, that the user can see the content. Model is an object model, which is used to respond to the corresponding specific operation and update the data of view. The relationship between the three is shown in Figure 2.5 below.

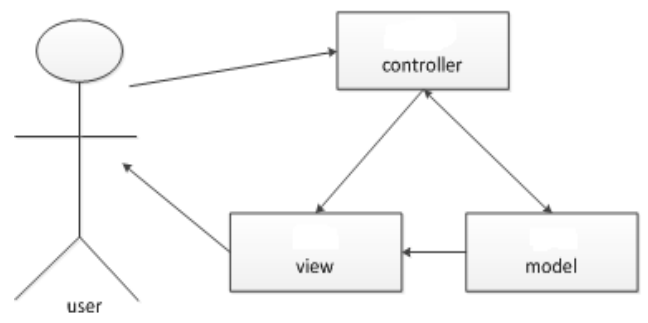

Fig.4 Schematic diagram of MVC

Model: Behavior and data encapsulation, rules and methods for modifying data and control behavior.

View: Model to show the graphics page, the user can see is the view, the user can also be carried out on the view of some operations.

Contmller: Connect the model and view, responsible for the data synchronization between the two, to accept the view of the operation information and feedback model changes.

\section{Struts}

Struts is an open source (OpenSource) project of the Apache software foundation Jakata project. Struts is widely used because of its simple and rapid development. Struts essentially a MVC model based on J2EE technology. Servlet and JSP are the main technologies used. The model helps developers to build multi tier applications more quickly, and improve the flexibility and reusability of modules. And the integrated technology is mature technology, learning and the use of low cost. Fig. 5 is the basic framework of the Struts.

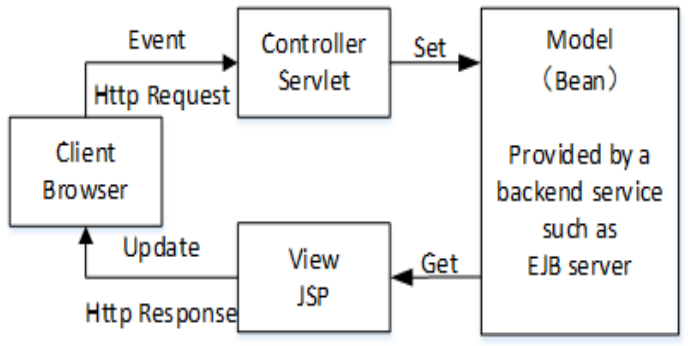

Fig.5 Struts basic framework

Struts provides support for the bottom of the MVC system, is a typical representation layer framework. Because of its JSP and HTML development completely separate characteristics, developers only need to pay attention to the realization of the function. With the help of a very strong expansion of the tag library, Struts can achieve complex business logic. Development 
efficiency based on Struts has been greatly improved. However, in view of the logical function, Struts does not have the good support, also is very difficult to carry on the logic reuse, therefore generally needs to combine other logical layers.

\section{Hibernate}

Itself is an independent framework for Java persistence, which does not require other things such as Server Web support. In the classic Web application, can be used as a data access layer, which can facilitate the use of Java to achieve the XML object or IO to the database of lasting impression. The use of the project development cycle can be shortened, only the use of data storage, do not need to understand the underlying implementation of the principle. HibernateIncluding Session, SessionFactory, Transaction, Query, Criteria and ConfigurationSix core interfaces. Access to persistent objects and transaction control are achieved through these six interfaces, the Fig.6 is a schematic diagram of the framework.

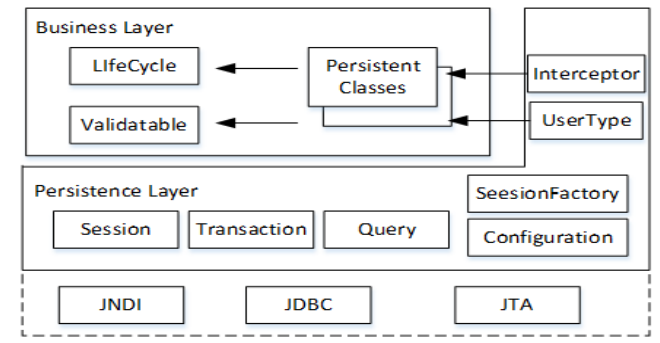

Fig.6 Hibernate frame diagram

\section{Spring}

Spring is a lightweight container that can be used to connect objects to the XML file. Object is a reference to a dependent object that can be obtained by viewing the properties of the object. XML configuration is used to connect these references.

The same Spring is a hierarchical structure that contains a total of seven modules . These modules can be arbitrarily combined or even exist alone. These modules are based on the core of the container, the management and configuration of Bean have the core container complete. Fig.7 is the basic framework of Spring

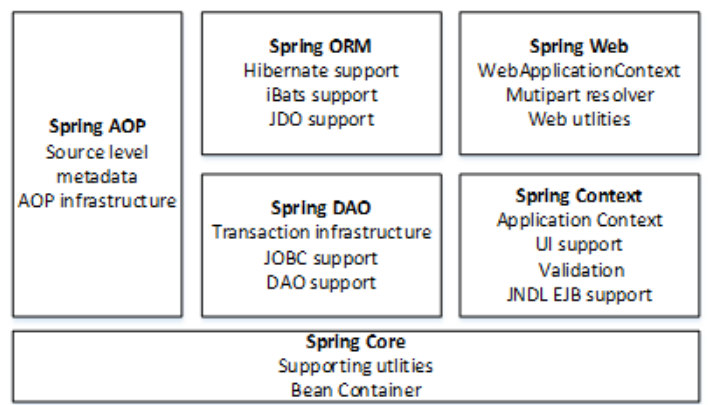

Fig.7 Basic framework of Spring

\section{Conclusion}

In this paper, we study the traffic monitoring system integrates the computer field of advanced technology, this chapter will mainly on the technology used in this paper were described in detail, including the J2EE technology and MVC technology and database storage technology.

\section{References:}

[1] Cho S, Quek C, Seah S, et al. HebbR2-Taffic: A novel application of neuro-fuzzy network for visual based traffic monitoring system[J]. Expert Systems with Applications, 2009, 
36(3):6343-6356.

[2] Rodríguez T, García N. An adaptive, real-time, traffic monitoring system[J]. Machine Vision and Applications, 2010, 21(4):555-576.

[3] Liao C, Iftode L, Nadeem T, et al. TrafficView: A Scalable Traffic Monitoring System.[J]. Mobile Data Management, Proceedings, IEEE International Conference on, 2004:13 - 26.

[4] Costea I M, Tanasa P A, Barbu I, et al. Automatic traffic monitoring system[C]. //Electronics Technology (ISSE), Proceedings of the 2014 37th International Spring Seminar on. IEEE, 2014:332 - 335.

[5] Zhenming W. Emergency Traffic Monitoring System for Urban Rail Transit[J]. Railway Signalling \& Communication Engineering, 2013.

[6] $\mathrm{H} \mathrm{H} \mathrm{W}$. The intelligent traffic monitoring system based on beidou research and design[J]. Applied Mechanics \& Materials, 2014.

[7] * X H Y, Guo S. The Design of Computer Network Traffic Monitoring System[J]. Modern Technologies in Materials, Mechanics and Intelligent Systems, 2014. 